\title{
Intervenções de enfermagem em cuidados paliativos na atenção primária à saúde:
}

\section{Scoping review}

\author{
Nursing interventions in palliative care in primary health care: Scoping review \\ Intervenciones de enfermería en cuidados paliativos en la atención primaria de salud: Scoping \\ review
}

\section{Resumo}

Objetivo: mapear as intervenções de enfermagem em cuidados paliativos na Atenção Primária à Saúde. Método: Trata-se de uma revisão de escopo. O instrumento PRISMA Extension for Scoping Review foi empregado para a redação do estudo. As buscas foram realizadas em seis bases de dados, a partir dos descritores cuidados paliativos, Atenção Primária à Saúde e cuidados de enfermagem. Resultados: Foram selecionados 23 estudos e, a partir deles, identificadas 63 intervenções de enfermagem, voltadas para: operacionalização dos cuidados paliativos na Atenção Primária à Saúde; promoção de uma assistência qualificada e integrada; família/cuidador no âmbito do domicílio; controle da dor; estabelecimento de uma comunicação eficiente e eficaz; e alterações psicossociais e espirituais. Conclusão: O mapeamento da produção científica mostrou que, embora, nos últimos anos, tenha havido avanços, as intervenções de enfermagem em cuidados paliativos na Atenção Primária à Saúde ainda têm sido implementadas de forma incipiente. Tal fato reflete na necessidade de uma capacitação profissional aprimorada com o intuito de ampliar o acesso aos cuidados paliativos e melhorar o serviço prestado.

Palavras-chave: Cuidados paliativos; Atenção primária à saúde; Cuidados de enfermagem; Enfermagem.

\begin{abstract}
Objective: to map nursing interventions in palliative care in primary health care. Method: This is a scope review. The PRISMA Extension for Scoping Review instrument was used for the writing of the study. Searches were performed in six databases, using the descriptors palliative care, primary health care and nursing care. Results: 23 studies were selected and, from them, 63 nursing interventions were identified, focused on: operationalization of palliative care in primary health care; promotion of qualified and integrated assistance; family/caregiver within the home; pain control; establishing efficient and effective communication; and psychosocial and spiritual changes. Conclusion: The mapping of scientific production showed that, although there have been advances in recent years, nursing interventions in palliative care in primary health care have still been implemented in an incipient way. This fact reflects the need for improved professional training in order to expand access to palliative care and improve the service provided.
\end{abstract}

Keywords: Palliative care; Primary health care; Nursing care; Nursing. 


\section{Resumen}

Objetivo: mapear las intervenciones de enfermería en cuidados paliativos en la atención primaria de salud. Método: esta es una revisión del alcance. Para la redacción del estudio se utilizó el instrumento PRISMA Extension for Scoping Review. Las búsquedas se realizaron en seis bases de datos, utilizando los descriptores cuidados paliativos, atención primaria de salud y atención de enfermería. Resultados: se seleccionaron 23 estudios y, de ellos, se identificaron 63 intervenciones de enfermería, enfocadas en: operacionalización de los cuidados paliativos en la atención primaria de salud; promoción de la asistencia calificada e integrada; familia / cuidador dentro del hogar; control de dolor; establecer una comunicación eficiente y eficaz; y cambios psicosociales y espirituales. Conclusión: El mapeo de la producción científica mostró que, si bien se han producido avances en los últimos años, las intervenciones de enfermería en cuidados paliativos en la atención primaria de salud aún se han implementado de manera incipiente. Este hecho refleja la necesidad de una mejor formación profesional para ampliar el acceso a los cuidados paliativos y mejorar el servicio prestado.

Palabras clave: Cuidados paliativos; Primeros auxilios; Cuidado de enfermera; Enfermería.

\section{Introdução}

A partir da Declaração de Alma-Ata em 1978, a Atenção Primária à Saúde (APS) se consolida como porta de entrada dos indivíduos, família e comunidade dentro dos sistemas de saúde, que deve promover e organizar a continuidade de assistência à saúde, com ações de promoção, de prevenção, de tratamento, de recuperação e de reabilitação (Portela, 2017).

Refletindo a organização dos sistemas de saúde mundiais, a APS brasileira articula-se aos demais níveis de atenção em arranjos organizacionais denominados Redes de Atenção à Saúde (RAS), que têm os seus princípios previstos pela Política Nacional de Atenção Básica (PNAB), sendo eles: acessibilidade, vínculo, coordenação, integralidade e longitudinalidade do cuidado (Brasil, 2017).

Em muitos países a APS é considerada o melhor nível de assistência à saúde para prestação da abordagem dos Cuidados Paliativos (CP), justificada principalmente pela proximidade emocional, cultural e geográfica dos profissionais inseridos neste contexto, contribuindo para que o cuidado seja humanizado, respeitando-se a autonomia da pessoa e de sua família e, evitando-se a fragmentação do indivíduo (Ribeiro \& Poles, 2019).

A APS permite ainda, que o indivíduo permaneça em casa, de forma a evitar seu afastamento da família nos momentos finais de vida (Ribeiro \& Poles, 2019). Ademais, por estar indicada desde o início das doenças graves e progressivas em conjunto com as terapias modificadoras de doença, a APS é o nível ideal de oferta dos CP, seja para cumprir o seu papel como ordenadora do sistema ou de ofertar cuidado integrado, desde o início do diagnóstico, ajudando a gerenciar as necessidades físicas, psicológicas e sociais dos pacientes e familiares (Roth \& Canedo, 2019).

As equipes dos serviços que compõem a APS devem ter formação multiprofissional. Contudo, é de responsabilidade do enfermeiro da unidade promover o gerenciamento, o planejamento e a avaliação das ações dos Agentes Comunitários de Saúde (ACS) agregados com os outros membros da equipe (Brasil, 2017).

Embora o enfermeiro exerça várias atividades de extrema importância para a qualidade do serviço prestado, é sabida a lacuna acerca de sua formação em relação aos $\mathrm{CP}$, devido à falta de capacitação e de treinamentos específicos para estes profissionais (Ikeda et al., 2017). Neste contexto, este estudo teve como objetivo mapear as evidências disponíveis acerca das intervenções de enfermagem a pacientes em CP na APS.

\section{Metodologia}

Trata-se de uma Revisão de Escopo conduzida a partir das etapas: (1) identificar a questão de pesquisa, (2) identificar estudos relevantes, (3) seleção de estudo, (4) mapear os dados e (5) comparar, sumarizar e descrever os resultados, conforme recomendações do Joanna Briggs Institute Reviewer's Manual (Peters et al., 2020). Este tipo de revisão possibilita a síntese de conhecimento sobre determinado assunto ao incorporar uma variedade de estudos com delineamentos diversos para resumir e 
sintetizar evidências de forma abrangente. Seus resultados podem contribuir para informar a prática, os programas e as políticas, além de fornecer orientação para futuras prioridades de pesquisa (Colquhoun et al., 2014). O relato desta revisão seguiu o proposto pelo PRISMA Extension for Scoping Reviews (PRISMA-ScR) (Tricco et al., 2018).

A busca foi norteada pela pergunta "Quais são as evidências científicas acerca das intervenções de Enfermagem utilizadas em pacientes em CP assistidos em serviços da APS?”. Esta questão foi construída a partir da estratégia mnemônica PCC (População, Conceito e Contexto) (Tricco et al., 2018): P - estudos envolvendo pacientes em CP; C - intervenções de enfermagem; $\mathrm{C}$ - serviços de APS.

As definições apresentadas a seguir guiaram a condução desta revisão, bem como as estratégias de busca: pacientes em CP (qualquer pessoa diagnosticada com uma condição limitante de vida e recebendo cuidados hospice ou serviço de cuidados paliativos); intervenções de Enfermagem (qualquer intervenção, baseada no julgamento e no conhecimento clínico, que seja realizada por um enfermeiro para melhorar os resultados do paciente) (Bulechek, Butcher \& Dochterman, 2016); serviços de APS (unidades de saúde da família; Núcleos de Apoio à Saúde da Família (NASF); Serviços de Atenção Domiciliar (SAD); hospice).

A busca dos estudos foi realizada de agosto a outubro de 2019 nas bases de dados CINAHL, MedLine, Lilacs, Scopus e Web of Science. Foram utilizados os seguintes Descritores Controlados em Ciências da Saúde (Decs) e Medical Subject Headings (MeSH), em português, inglês e espanhol: 'Atenção Primária à Saúde'; 'Cuidados de Enfermagem'; 'Cuidados Paliativos'; 'Primary Health Care'; 'Nursing Care'; 'Palliative Care'; 'Atención Primaria de Salud'; 'Atención de Enfermería'; 'Cuidados Paliativos'. Para efetuar o cruzamento dos descritores, foi utilizado o operador booleano AND.

Os critérios de elegibilidade foram: artigos científicos disponibilizados na íntegra; nos idiomas inglês, português ou espanhol; publicados no período de 2009 a 2019, cujos títulos e resumos contemplassem os termos 'cuidados paliativos', 'atenção primária à saúde' e ‘cuidados e/ou intervenções de enfermagem'. Foram excluídas cartas ao editor, editoriais e artigos de opinião. O estabelecimento do recorte temporal como um dos critérios de elegibilidade justifica-se pela intensificação da produção científica sobre $\mathrm{CP}$ neste período.

Os resultados das buscas foram exportados para o EndNote e os artigos duplicados removidos. Primeiramente, foi feita a leitura dos títulos e resumos e, posteriormente, os artigos pré-selecionados foram lidos na íntegra, a fim de se excluir aqueles que não atendiam aos critérios de elegibilidade. A seleção dos estudos foi realizada por dois revisores independentes e devidamente capacitados para a atividade, de modo que as incongruências foram discutidas e acordadas. Quando necessário, um terceiro revisor foi envolvido para a resolução das discordâncias.

A extração de dados foi realizada por dois pesquisadores independentes e treinados para tal atividade. Utilizou-se um instrumento desenvolvido pelos autores para coleta dos seguintes dados: identificação do estudo (ano e periódico de publicação, país onde o estudo foi realizado), objetivo, metodologia, população, intervenções de enfermagem aplicadas a pessoas em CP na APS, principais resultados e conclusões.

Os dados foram analisados a partir de três questões interativas: "O que os dados estão me dizendo?"; "O que nós queremos saber?"; "Qual é a relação dialética entre os que os dados estão me dizendo e o que quero saber?" (Srivastava \& Hopwood, 2009). Esta etapa foi feita pelos mesmos pesquisadores que realizaram a abordagem do estudo de "peneirar, mapear e classificar o material, de acordo com as principais questões e temas" (Arksey \& O'Malley, 2005). As descobertas resultantes desse processo foram revisadas e refinadas em colaboração com um terceiro pesquisador. Posteriormente, os estudos foram comparados quanto aos achados comuns e recorrentes em relação as intervenções de enfermagem para o estabelecimento de semelhanças e de diferenças, e os dados foram organizados em categorias temáticas (Arksey \& O'Malley, 2005; Levac, Colquhoun, \& O'Brien, 2010).

Para a apresentação dos dados, optou-se por construir um quadro que procurou apresentar as intervenções de 
enfermagem apontadas pelos diferentes estudos.

\section{Resultados}

A amostra desta revisão foi composta por 23 estudos. O processo de seleção e inclusão dos artigos está apresentado na Figura 1.

Figura 1 - Processo de identificação e inclusão dos estudos - Preferred Reporting Items for Systematic re- views and MetaAnalyses extension for Scoping Reviews (PRISMA-ScR) Checklist, Alfenas, MG, Brasil, 2020.

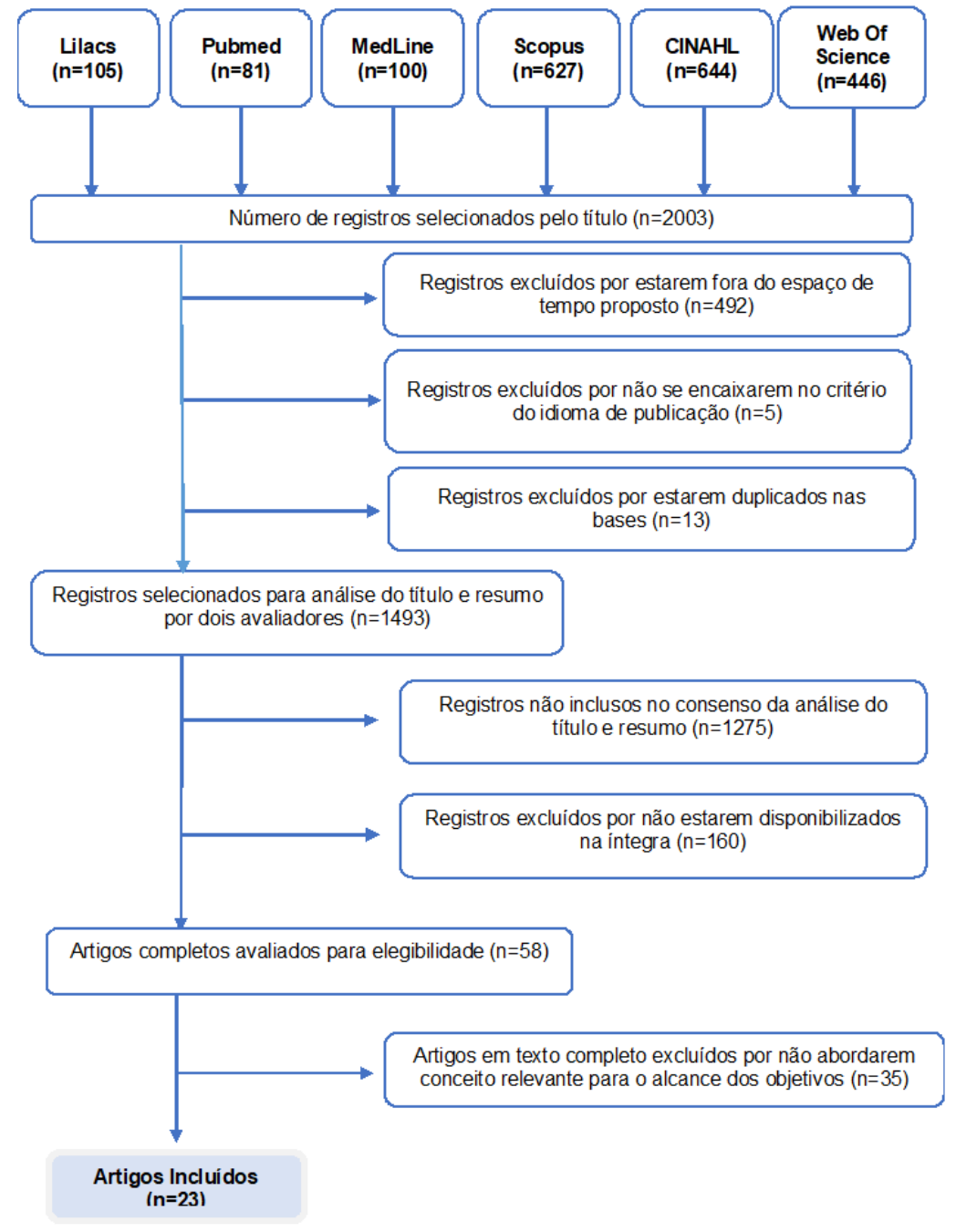

Fonte: Dados da Pesquisa (2020).

A partir dos artigos que compõem a amostra, foi possível extrair intervenções de enfermagem em CP na APS (Quadro 1). É oportuno assinalar que, de acordo com a Classificação das Intervenções de Enfermagem (NIC), as intervenções de enfermagem incluem cuidado direto e indireto. Em relação ao cuidado direto, este inclui as ações de enfermagem fisiológicas e psicológicas. Enquanto a intervenção de cuidado indireto, contempla o tratamento realizado longe do paciente, mas favorecendo-o ou ao grupo de pacientes. Portanto, incluem ações dirigidas ao gerenciamento do ambiente de cuidado do paciente e da colaboração multidisciplinar (Bulechek, Butcher \& Dochterman, 2016). Por conseguinte, as intervenções de enfermagem assinaladas nesse estudo foram construídas com o escopo de abranger os cuidados diretos e indiretos, conforme prescritos na NIC. 
Quadro 1 - Intervenções de enfermagem em CP na APS, dispostas pelos estudos inclusos na Revisão de Escopo. Alfenas, Minas Gerais, Brasil, 2020.

\begin{tabular}{|c|c|}
\hline Categoria & Intervenções de Enfermagem \\
\hline \multirow{12}{*}{$\begin{array}{l}\text { Intervenções voltadas } \\
\text { para a } \\
\text { operacionalização dos } \\
\text { CP na APS }\end{array}$} & $\begin{array}{l}\text { Operar na organização, análise e melhoria dos cuidados de saúde em domicílio (Sousa \& Alves, 2015; } \\
\text { Pereira et al., 2017). }\end{array}$ \\
\hline & Viabilizar a coordenação do cuidado (Silva, 2013). \\
\hline & Buscar auxílio na resolutividade de problemas (Lai, Wong \& Ching, 2018). \\
\hline & $\begin{array}{l}\text { Identificar barreiras e ações facilitadoras para pacientes e cuidadores na utilização eficaz de recursos em } \\
\text { domicílio (Sousa \& Alves, 2015; Lai, Wong \& Ching, 2018). }\end{array}$ \\
\hline & $\begin{array}{l}\text { Desenvolver propostas para melhorar o atendimento por meio da busca de soluções destinadas a prevenir } \\
\text { a fragmentação do atendimento (Nevado, Babarro \& Lozano, 2012). }\end{array}$ \\
\hline & Constatar mudanças dinâmicas na demografia da população (Sousa \& Alves, 2015). \\
\hline & Facilitar o acesso aos CP pela família e paciente (Silva, 2013). \\
\hline & Avaliar o impacto do atendimento prestado (Fitch, Fliedner \& O'Connor, 2015). \\
\hline & Buscar aprimoramento profissional (Fitch, Fliedner \& O'Connor, 2015). \\
\hline & Estabelecer projetos de investigação em cuidados paliativos (Sousa \& Alves, 2015). \\
\hline & Saber se adequar ao local de trabalho (Picollo \& Fachini, 2019). \\
\hline & Mobilizar recursos sociais (Nevado, Babarro \& Lozano, 2012). \\
\hline \multirow{17}{*}{$\begin{array}{l}\text { Intervenções voltadas } \\
\text { para a promoção de } \\
\text { uma assistência } \\
\text { qualificada e } \\
\text { integrada }\end{array}$} & Garantir assistência com integralidade (Silva, 2013; Picollo \& Fachini, 2019). \\
\hline & Utilizar a longitudinalidade como ferramenta de cuidado (Silva, 2013). \\
\hline & $\begin{array}{l}\text { Conjugar o paciente e sua família como foco de cuidado da equipe multiprofissional (Sousa \& Alves, } \\
2015 \text { ). }\end{array}$ \\
\hline & $\begin{array}{l}\text { Elaborar planos de cuidados avançados em cima das necessidades dos pacientes e família/cuidadores, } \\
\text { buscando avaliar complicações de comprometimento cognitivo, risco de quedas, status de vacinação e } \\
\text { triagem de causas reversíveis de desnutrição (Sousa \& Alves, 2015; Wenger et al., 2011; Lokker et al., } \\
\text { 2018; Pirret, Neville \& La Grow, 2015; Cleary, 2016). }\end{array}$ \\
\hline & Estabelecimento de vínculo e da confiança paciente/enfermeiro (Sousa \& Alves, 2015). \\
\hline & $\begin{array}{l}\text { Realizar monitoramento e avaliação contínuos dos planos de intervenção realizados (Nevado, Babarro \& } \\
\text { Lozano, 2012). }\end{array}$ \\
\hline & Fornecer intervenções baseadas em evidências (Fitch, Fliedner \& O'Connor, 2015). \\
\hline & Identificar e intervir em caso de baixa adesão aos medicamentos (Pirret, Neville \& La Grow, 2015). \\
\hline & Promover conforto em prol da sedação paliativa (Lokker et al., 2018). \\
\hline & $\begin{array}{l}\text { Operar na avaliação, manejo e controle de sinais e sintomas comuns no final da vida, por meio de } \\
\text { instrumentos padronizados (Sousa \& Alves, 2015; Fitch, Fliedner \& O'Connor, 2015; Dahlin, Coyne \& } \\
\text { Cassel, 2016). }\end{array}$ \\
\hline & $\begin{array}{l}\text { Avaliar o impacto das terapias tradicionais, integrativas, complementares e tecnológica centrada em } \\
\text { resultados dos pacientes (Sousa \& Alves, 2015). }\end{array}$ \\
\hline & $\begin{array}{l}\text { Identificar a iminência da morte e empregar cuidados adequados ao paciente e família, proporcionando } \\
\text { conforto e dignidade (Sousa \& Alves, 2015). }\end{array}$ \\
\hline & $\begin{array}{l}\text { Estabelecer com pacientes e cuidadores um plano de atividade física que favoreça a mobilidade no } \\
\text { domicílio (Sousa \& Alves, 2015; Brant, Fink \& Thompson, 2019). }\end{array}$ \\
\hline & Trabalhar com parcerias dentro e fora da APS (Silva, 2013). \\
\hline & $\begin{array}{l}\text { Empregar os postulados éticos dos cuidados paliativos na tomada de decisão em questões complexas de } \\
\text { fim de vida, reconhecendo a influência dos valores pessoais, código de ética profissional e preferências } \\
\text { do paciente (Sousa \& Alves, 2015). }\end{array}$ \\
\hline & Promover disseminação de informações dentro da equipe (Mertens et al., 2019). \\
\hline & Definição clara de papeis dentro da equipe (Van der Plas et al., 2016). \\
\hline \multirow{12}{*}{$\begin{array}{l}\text { Intervenções voltadas } \\
\text { para a } \\
\text { família/cuidador no } \\
\text { âmbito do domicílio }\end{array}$} & Responsabilizar-se pelo cuidado paliativo com a família (Silva, 2013). \\
\hline & Promover acolhimento à família (Picollo \& Fachini, 2019). \\
\hline & $\begin{array}{l}\text { Estabelecer e executar com cuidadores que apresentam risco de distress ou sobrecarga, um plano } \\
\text { compartilhado de cuidados domiciliares (Griffiths et al., 2015). }\end{array}$ \\
\hline & $\begin{array}{l}\text { Executar educação de familiares e cuidadores para avaliação e manejo de sinais e sintomas em domicílio } \\
\text { comuns no final da vida (Sousa \& Alves, 2015). }\end{array}$ \\
\hline & $\begin{array}{l}\text { Orientar pacientes e familiares acerca do processo de doença do paciente em final de vida (Sousa \& } \\
\text { Alves, 2015). }\end{array}$ \\
\hline & Trabalhar a tolerância e a aceitação dentro do âmbito domiciliar (Danielsen et al., 2018). \\
\hline & Estimular a participação da família (Holmdahl, Sävenstedt \& Imoni, 2014). \\
\hline & $\begin{array}{l}\text { Servir de elo entre família/paciente e equipe multiprofissional (Fitch, Fliedner \& O'Connor, 2015; Lokker } \\
\text { et al., 2018). }\end{array}$ \\
\hline & $\begin{array}{l}\text { Empregar com a equipe multiprofissional orientações a familiares sobre rituais de funeral, direitos sociais } \\
\text { e responsabilidades com papéis e documentos em situação de óbito (Sousa \& Alves, 2015). }\end{array}$ \\
\hline & $\begin{array}{l}\text { Oportunizar o acesso de familiares e cuidadores à equipe multiprofissional no período de luto (Sousa \& } \\
\text { Alves, 2015). }\end{array}$ \\
\hline & Colaborar com cuidadores informais (Van der Plas et al., 2016). \\
\hline & Valorizar os pontos de vista e desejos do paciente e família durante os cuidados em fim de vida ((Sousa \& \\
\hline
\end{tabular}




\begin{tabular}{|c|c|}
\hline \multirow{6}{*}{$\begin{array}{l}\text { Intervenções voltadas } \\
\text { para o controle da } \\
\text { dor }\end{array}$} & Alves, 2015; Graaf, 2016). \\
\hline & Promover manejo da dor (Dahlin, Coyne \& Cassel, 2016). \\
\hline & Avaliar nível de dor (Stenner, Carey \& Courtenay, 2012). \\
\hline & $\begin{array}{l}\text { Ficar atento a necessidade de nova avaliação médica para prescrição de medicamentos (Stenner, Carey \& } \\
\text { Courtenay, 2012). }\end{array}$ \\
\hline & Avaliar uso correto das medicações prescritas (Stenner, Carey \& Courtenay, 2012). \\
\hline & Administrar medicação para controle da dor em domicílio (Brant, Fink \& Thompson, 2019). \\
\hline \multirow{8}{*}{$\begin{array}{c}\text { Intervenções voltadas } \\
\text { para o } \\
\text { estabelecimento de } \\
\text { uma comunicação } \\
\text { eficiente e eficaz }\end{array}$} & Utilizar da habilidade da comunicação (Griffiths et al., 2015). \\
\hline & Promover uma escuta terapêutica (Griffiths et al., 2015). \\
\hline & Ouvir e interpretar necessidades (Picollo \& Fachini, 2019). \\
\hline & Usar dialeto compreensível ao paciente e familiares (Griffiths et al., 2015). \\
\hline & Fornecer informações relevantes, claras e autorizadas (Watts, 2014). \\
\hline & Manter boa comunicação com o serviço hospitalar de referência (Danielsen et al., 2018). \\
\hline & Avaliar o momento correto para informar más notícias (Griffiths et al., 2015). \\
\hline & $\begin{array}{l}\text { Expressar de forma eficaz com a comunidade e equipe de saúde sobre questões de fim de vida (Sousa \& } \\
\text { Alves, 2015). }\end{array}$ \\
\hline \multirow{8}{*}{$\begin{array}{l}\text { Intervenções frente as } \\
\text { alterações } \\
\text { psicossociais e } \\
\text { espirituais, com } \\
\text { ênfase no processo de } \\
\text { luto }\end{array}$} & $\begin{array}{l}\text { Promover apoio emocional durante a assistência ao paciente, à família, cuidadores e equipe de saúde, } \\
\text { incluindo o processo de luto (Sousa \& Alves, 2015; Lai, Wong \& Ching, 2018; Fitch, Fliedner \& } \\
\text { O'Connor, 2015; Brant, Fink \& Thompson, 2019; Griffiths et al., 2015). }\end{array}$ \\
\hline & $\begin{array}{l}\text { Monitorar e promover a detecção precoce de distúrbios psicossociais (Sousa \& Alves, 2015; Watts, } \\
\text { 2014). }\end{array}$ \\
\hline & $\begin{array}{l}\text { Encaminhar pacientes e familiares para participar de programas de apoio psicossocial e espiritual (Sousa } \\
\& \text { Alves, 2015). }\end{array}$ \\
\hline & $\begin{array}{l}\text { Compor a equipe multiprofissional na avaliação e manejo das necessidades psicossociais e espirituais } \\
\text { complexas do paciente e sua família (Sousa \& Alves, 2015). }\end{array}$ \\
\hline & Promover empatia (Griffiths et al., 2015). \\
\hline & $\begin{array}{l}\text { Identificar e lidar com o sofrimento existencial, emocional e espiritual (Lokker et al., 2018; Tornøe et al., } \\
\text { 2015). }\end{array}$ \\
\hline & $\begin{array}{l}\text { Interpretar as atitudes próprias, sentimentos, valores e expectativas sobre a morte, respeitando a } \\
\text { diversidade cultural e espiritual (Sousa \& Alves, 2015). }\end{array}$ \\
\hline & Promover a auto-ajuda do paciente/família (Nevado, Babarro \& Lozano, 2012). \\
\hline
\end{tabular}

Fonte: Dados da Pesquisa (2020).

\section{Discussão}

O estudo se propôs a fazer um mapeamento das evidências disponíveis acerca das intervenções de enfermagem a pacientes em CP na APS verificando que, embora nos últimos anos tenha havido avanços, as intervenções de enfermagem em $\mathrm{CP}$ no referido contexto ainda têm sido implementadas de forma incipiente.

No que tange a categoria "Intervenções voltadas para a operacionalização dos CP na APS", percebe-se a promoção de ações que facilitem, avaliem, auxiliem e aprimorem os CP na APS, incluindo também, o contexto do âmbito domiciliar (Sousa \& Alves, 2015; Pereira et al., 2017). Dentre essas ações, a produção destaca a coordenação do cuidado (Silva, 2013). Segundo uma pesquisa realizada em 2017, essa coordenação é capaz de atender as necessidades dos usuários do sistema de saúde em sua totalidade (Almeida, Marin \& Casotti, 2017).

Outra intervenção incluída na respectiva categoria, refere-se à resolutividade de problemas (Lai Wong \& Ching, 2018). O estudo assevera que essa resolutividade possibilita uma assistência qualificada dentro dos CP, culminando em melhores achados clínicos, sendo possível obter resultados financeiros positivos (Hawkins et al., 2015). A pesquisa ratifica a importância de ser hábil para resolver problemas, a fim de favorecer a qualidade do serviço (Lai Wong \& Ching, 2018).

Um estudo reforça a relevância de melhorar o atendimento prestado através de soluções destinadas a prevenir fragmentações (Nevado, Babarro \& Lozano, 2012). Uma das maiores dificuldades na prestação de serviço em CP de boa qualidade é a falta de continuidade na assistência que associado a baixa disponibilidade de recursos, torna-se um fator negativo de grande importância (Midtbust et al., 2018).

Outra intervenção aqui citada, é em relação a magnitude de se constatar mudanças na dinâmica demográfica da população assistida (Sousa \& Alves, 2015). É importante ter a dimensão de quem está sendo assistido pela APS, a fim de 
identificar barreiras de acesso ao serviço prestado e proporcionar o cuidado centralizado na pessoa e em suas peculiaridades dentro do âmbito socioeconômico, respeitando suas culturas e fatores limitantes (Elk et al., 2018).

Um estudo internacional chama atenção para o que se refere ao processo de triagem realizado pelos profissionais de enfermagem para nortear as estratégias em CP (Sousa \& Alves, 2015). Destaca-se que o levantamento de informações através desse processo de triagem é de suma importância para identificar a elegibilidade de pessoas aos CP. Todavia, esta inferência não foi contemplada nos estudos analisados nesta revisão, bem como o emprego de escalas utilizadas para esta avaliação, tais como: Escala de Performance Paliativa (PPS) (Campos et al., 2009); Escala de Performance de Karnofsky (KPS) (Marcucci et al., 2016); Supportive and Palliative Care Indicators Tool (SPICT-DE) (Afshar et al., 2020).

Constatou-se na produção científica que um fator primordial quando se diz respeito a melhoria do serviço de enfermagem em CP é o aprimoramento profissional (Silva, 2013). Com o aumento da demanda pelos CP, percebe-se a escassez de profissionais capacitados na área. O aprimoramento profissional de enfermeiros especializados em CP favorece a assistência centrada na pessoa e, consequentemente, proporciona serviços de qualidade (Levine et al., 2017).

Quanto a categoria "Intervenções voltadas para a promoção de uma assistência qualificada e integrada", nota-se uma grande preocupação dos estudos brasileiros em garantir e consolidar os princípios do sistema brasileiro de saúde denominado Sistema Único de Saúde (SUS) para a assistência em CP, a saber: integralidade (saúde voltada para a inserção social, o indivíduo inserido em uma comunidade e como isso influencia no processo de saúde-doença), universalidade (garante acesso universal à saúde) e equidade (acesso de maneira igualitária, sem privilégios ou preconceitos) (Frazzatto \& Sawaia, 2016). Destaca-se que a preocupação dos estudos em promover uma assistência qualificada e integrada pode estar alinhada ao fato de que o SUS ainda não está consolidado (Paim, 2018). Além disso, no Brasil a Resolução No 41/2018, que dispõe sobre as diretrizes para a organização dos CP, à luz dos cuidados continuados integrados, no âmbito do SUS, é extremamente recente.

Segundo o estudo, garantir uma assistência com integralidade reverbera a preocupação dos autores com o risco da fragmentação da assistência de pacientes em $\mathrm{CP}$, em virtude ao grande número de especialistas e de serviços de urgência/emergência, que acompanham esses casos ofertando assistência pontual diante de condições agudas ou de exacerbação da doença principal, sem a garantia de continuidade do cuidado dentro do sistema de saúde (Silva, 2013).

Nesse sentido, conforme aponta a pesquisa, empregar a longitudinalidade como ferramenta de cuidado as pessoas em CP, caracteriza-se como mais uma potencialidade da APS (Silva, 2013). Esta se consolida por meio da existência de uma fonte habitual de atenção e o seu emprego ao longo do tempo, partindo da premissa de que a relação entre os usuários e os profissionais deve refletir confiança interpessoal e um vínculo duradouro (Harzheim et al., 2016). Este estabelecimento de vínculo e da confiança paciente/enfermeiro foi apontado no estudo analisado por esta revisão (Sousa \& Alves, 2015).

Logo, a manutenção do acompanhamento por profissionais da APS, em destaque o enfermeiro, aos indivíduos em CP, proporcionaria um cuidado integral, com ações de promoção em saúde, além da redução de uso de serviços de alta complexidade com a diminuição de custos no setor da saúde. Tais achados vão ao encontro da intervenção apontada na pesquisa ao mencionar que a enfermagem deve conjugar o paciente e sua família como foco de cuidado da equipe multiprofissional (Sousa \& Alves, 2015).

Constatou-se nos estudos analisados que para uma assistência de enfermagem adequada à pessoa em $\mathrm{CP}$, faz-se necessário a elaboração de planos de cuidados avançados acerca das necessidades dos pacientes e família/cuidadores. Estes planos devem contemplar as seguintes intervenções: identificar a iminência da morte e empregar cuidados adequados ao paciente e família, proporcionando conforto e dignidade; identificar e intervir em caso de baixa adesão aos medicamentos; avaliar complicações de comprometimento cognitivo, risco de quedas, status de vacinação e triagem de causas reversíveis de desnutrição; operar na avaliação, manejo e controle de sinais e sintomas comuns no final da vida, por meio de instrumentos 
padronizados; avaliar o impacto das terapias tradicionais, integrativas, complementares e tecnológicas centradas nos resultados dos pacientes; promover conforto em prol da sedação paliativa; estabelecer com pacientes e cuidadores um plano de atividade física que favoreça a mobilidade no domicílio; e realizar monitoramento e avaliação contínuos dos planos de intervenção realizados (Wenger et al., 2011; Sousa \& Alves, 2015; Pirret, Neville \& La Grow, 2015; Cleary, 2016; Lokker et al., 2018).

Adicionalmente, para a consolidação desses planos de cuidados é de fundamental importância que os enfermeiros trabalhem com parcerias dentro e fora da APS (Silva, 2013), buscando promover disseminação de informações dentro da equipe, além de uma definição clara de papéis dentro da equipe multiprofissional (Van der Plas et al., 2016).

Nos referidos planos de cuidados, conforme abordam os estudos da amostra, as intervenções devem ser baseadas em evidências (Wenger et al., 2011; Sousa \& Alves, 2015; Pirret, Neville \& La Grow, 2015; Cleary, 2016; Lokker et al., 2018). A produção assevera que a Prática Baseada em Evidências (PBE) caracteriza-se como forma segura e organizada de estabelecer condutas profissionais (Karnick, 2016). É reconhecido que a adesão PBE reduz o custo dos cuidados e melhora os resultados de saúde (Hweidi et al., 2017).

Ainda em relação a assistência de enfermagem ao paciente em CP, o estudo chama a atenção para a necessidade de empregar os princípios éticos dos CP na tomada de decisão em questões complexas de fim de vida, atentando-se ao código de ética profissional, reconhecendo a influência dos valores pessoais e as preferências da pessoa (Sousa \& Alves, 2015).

É nesse contexto que os enfermeiros da APS necessitam, ao cuidar de pacientes em CP, conhecer profundamente a história de vida e dos valores de cada indivíduo e, dentro do possível, estabelecer as Diretivas Antecipadas de Vontade (DAV). Estas objetivam manifestar a vontade da pessoa a respeito de cuidados, tratamentos e intervenções às quais deseja (ou não) ser submetida quando estiver impossibilitada de se expressar (Omondi et al., 2017). Ressalta-se que as diretivas podem ser revogadas a qualquer momento pela pessoa, mas, caso não o faça, entrarão em vigor quando este se tornar incapaz de tomar as próprias decisões (Dadalto, 2015).

Diante de tais ponderações, infere-se que os planos de cuidados de enfermagem voltados para o paciente em CP, no âmbito da APS, devem contemplar intervenções com vistas a promover conforto, associado ao alívio da dor, aproximação com entes queridos, promoção de dignidade e respeito.

Em relação à categoria "Intervenções voltadas para a família/cuidador no âmbito do domicílio", observa-se o importante papel da família durante o processo de progressão dos CP dentro do âmbito domiciliar. A demanda da necessidade de CP tem crescido em larga escala e, com ela, o aumento da participação de familiares e cuidadores informais é de grande importância para os bons resultados na assistência prestada ao paciente (Ullrich et al., 2017). A produção científica da amostra traz cuidadores informais e familiares como aliados quanto ao manejo de sinais e sintomas, uma vez que, estes por estarem em contato contínuo com o paciente, são aqueles que podem identificar alterações no quadro clínico com maior facilidade quando instruídos adequadamente (Sousa \& Alves, 2015).

$\mathrm{O}$ estudo destaca o profissional de enfermagem como personagem capaz de identificar desconfortos biopsicossociais em familiares e cuidadores informais, e de propor planos para aliviar a situação (Griffiths et al., 2015). A pesquisa enfatiza a necessidade de o profissional de enfermagem servir de elo entre paciente, família e equipe multiprofissional (Lokker et al., 2018). Ademais, é importante manter paciente e familiares informados a respeito da evolução da enfermidade desde o diagnóstico, além de tornar a comunicação uma aliada, visto que, essa pode amenizar sofrimentos, tanto durante o processo de adoecimento quanto na fase do luto (ANCP, 2020).

O estudo mostra a assistência em CP no processo do luto tão importante quanto as ações durante o processo de adoecimento, tanto em questões emocionais quanto burocráticas como funeral, direitos e documentos (Sousa \& Alves, 2015). 
Tais achados vão ao encontro do artigo ao afirmar que a assistência de enfermagem facilita as transições entre as fases dos CP para os entes durante a evolução do enfermo, inclusive durante o processo de luto (Hudson et al., 2019).

Por conseguinte, a avaliação e o tratamento do luto devem se fazer presente nas intervenções em CP para pacientes na terminalidade da vida, sendo necessário, a avaliação e o gerenciamento adequados de problemas emocionais, levando em consideração as necessidades dos familiares. Ressalta-se que o apoio em favor dos familiares deve ser realizado em períodos prévios e posteriores à morte do ente querido (Fox, Azman \& Timmons, 2020).

Em relação à categoria, "Intervenções frente as alterações psicossociais e espirituais", foi notório nos estudos da amostra que a enfermagem deve compor a equipe multiprofissional para realização da avaliação e do manejo das necessidades psicossociais e espirituais complexas do paciente em CP e sua família (Watts, 2014; Sousa \& Alves, 2015; Fitch, Fliedner \& O'Connor, 2015; Griffiths et al., 2015; Wong \& Ching, 2018; Brant, Fink \& Thompson, 2019). É de fundamental importância o papel do enfermeiro para a avaliação dos aspectos psicossociais do paciente, com vistas a identificar as fontes de sofrimento, bem como para ajudar a implementar um plano de minimização das dificuldades.

Dessa forma, os estudos da amostra apontam como intervenção de enfermagem o monitoramento e a detecção precoce de distúrbios psicossociais (Watts, 2014; Sousa \& Alves, 2015), sendo os mais comuns, a depressão (Hernando, Limonero \& Gil, 2020) e a ansiedade (Van den Brekel et al., 2020). O sofrimento também surge por meio de sentimentos de desesperança, desmoralização ou perda de controle (Warth et al., 2019).

Cabe ao enfermeiro realizar uma avaliação holística do paciente sob os $\mathrm{CP}$, com um olhar diferenciado, inclusive para o meio familiar, uma vez que esses distúrbios psicossociais promovem um sentimento de impotência no paciente e nos familiares, sendo assim necessário estimular o vínculo entre as partes (Borba et al., 2019). Após essa avaliação minuciosa e holística, é de suma importância que os enfermeiros encaminhem os pacientes e seus familiares para participarem de apoio psicossocial (Sousa \& Alves, 2015), os quais irão utilizar abordagens sofisticadas baseadas em evidências para lidar com o sofrimento clinicamente significativo (Ann-Yi et al., 2018).

$\mathrm{O}$ estudo proveniente desta revisão aponta como intervenção de enfermagem aos pacientes sob os $\mathrm{CP}$, o estabelecimento de uma relação empática, sendo de suma importância este cuidado ser pautado no estabelecimento de vínculos entre o profissional e o paciente (Griffiths et al., 2015). Outra intervenção de enfermagem contemplada neste estudo, diz respeito a identificação do sofrimento espiritual (Tornøe et al., 2015; Lokker et al., 2018). A pesquisa indica que um número significativo de pacientes em CP anseia por cuidado espiritual e existencial adequado (Tornøe et al., 2015). É oportuno assinalar que o cuidado espiritual é um componente importante do cuidado de saúde de alta qualidade, especialmente para pacientes em CP e suas famílias.

Diante de tais ponderações, infere-se que as intervenções de enfermagem aos pacientes em CP no contexto da APS, devem promover o bem-estar espiritual dos pacientes e de seus familiares, com vistas ajudar a desenvolver um senso de significado na vida e a experiência de paz, resultando em benefícios substanciais para a melhoria da saúde mental no final da vida. Além dos distúrbios psicossociais e espirituais, é de fundamental importância que os profissionais de enfermagem busquem o controle da dor dos pacientes em CP.

Na categoria "Intervenções voltadas para o controle da dor", observou-se que três estudos citaram como uma das intervenções, a promoção do manejo da dor, sendo esta elencada como o primeiro princípio dos CP, segundo a OMS: "a promoção do alívio da dor e outros sintomas desagradáveis" (Stenner, Carey \& Courtenay, 2012; Silva, 2013; Brant, Fink \& Thompson, 2019).

Ainda assim, observa-se que o reconhecimento e o manejo da dor na fase final de vida permanecem como uma problemática (Coyne, Mulvenon \& Paice, 2018). Sob a ótica dos pacientes e dos familiares, a dor é vista como o sintoma mais temido no processo de adoecer (Barros et al., 2020). Segundo uma revisão de literatura realizada em 2016, a definição de uma 
boa morte para $81 \%$ dos entrevistados, que incluíram pacientes, familiares e profissionais de saúde, é um "estado sem dor" (Meier et al., 2016).

Desse modo, corroborando com o resultado obtido no presente estudo, o manejo da dor é identificado como essencial no fim da vida (Virdun et al., 2017). Segundo a Sociedade Americana de Enfermagem (2017) se tratando de manejo clinicamente excelente da dor, deve observar "as indicações clínicas, a identificação mútua de metas para o manejo da dor, a colaboração interprofissional e o conhecimento dos padrões profissionais para a avaliação e o tratamento de diferentes tipos de dor". Entende-se que é indissociável o controle da dor no cenário dos $\mathrm{CP}$, em que o profissional de enfermagem, particularmente, participa ativamente, objetivando a melhoria da qualidade de vida.

Como observado entre os resultados aqui apresentados, o estudo referiu que a enfermagem deve se atentar para a avaliação do nível de dor, e na necessidade de nova avaliação médica para prescrição de medicamentos, com intervenções pertinentes (Stenner, Carey \& Courtenay, 2012). As produções apresentam uma lista de barreiras para o manejo ideal da dor na fase final de vida, dentre elas, a avaliação inadequada da dor, que abrange vários aspectos, tais como: negação da dor, não utilização de escalas de avaliação específicas para o público em questão e desconhecimento a respeito da dor total, ignorando os âmbitos psicológicos, sociais, culturais e espirituais (Mayahara et al., 2015; Gunnarsdottir et al., 2017).

Constata-se que a utilização de ferramentas multidimensionais, que abarquem todos os aspectos que envolvem a experiência dolorosa, uma avaliação acurada, completa e sistemática da dor em CP é crucial para orientação terapêutica. Vale destacar que a falta de acesso as medicações analgésicas, especialmente em países de baixa e média renda, é também uma das barreiras para o controle eficaz desse sintoma (Knaul et al., 2018).

Os estudos elencaram como intervenções, a administração de medicação para controle da dor em domicílio e a avaliação do uso correto das medicações prescritas, temáticas pouco exploradas na literatura (Stenner, Carey \& Courtenay, 2012; Brant, Fink \& Thompson, 2019).

No que diz respeito às "Intervenções voltadas para o estabelecimento de uma comunicação eficiente e eficaz”, o trabalho destaca a necessidade de implementar intervenções que sejam permeadas pela utilização da habilidade de comunicação (Holmdahl, Sävenstedt \& Imoni, 2014). Confluindo com o que foi observado no presente estudo, já foi visto que há forte correlação entre habilidades de comunicação dos enfermeiros com o bem-estar geral e a satisfação das pessoas com câncer (Baer \& Weinstein, 2013). Assim, a capacidade dos enfermeiros para comunicação em CP não é necessária apenas para fornecer informações, mas também, interfere diretamente em diversos âmbitos, como o planejamento dos cuidados, o manejo dos sintomas, o apoio psicológico, social e espiritual

Com vistas à importância das habilidades de comunicação no contexto dos $\mathrm{CP}$ e o aprimoramento dos enfermeiros nesse sentido, o Instituto Nacional do Câncer dos Estados Unidos da América financiou uma pesquisa que subsidiou a elaboração do COMFORT, um currículo de treinamento de comunicação em CP para enfermeiros (Wittenberg \& Goldsmith, 2016). Estudos promovidos com enfermeiros sobre esta ferramenta demonstraram que estes obtiveram melhora na autoeficácia clínica, na comunicação em si e no nível de conforto e de confiança em relação as conversas em CP, com pacientes, familiares e cuidadores (Cronin \& Finn, 2017).

Conforme assinala o COMFORT, o primeiro diálogo com o paciente e seus familiares é de suma importância, haja vista que vai fornecer subsídios para adaptação da comunicação, de modo que esta seja apropriada e eficaz e ainda, embasar a elaboração do plano de cuidados singular, o que corrobora com as seguintes intervenções: promover uma escuta terapêutica e interpretar necessidades elencadas nos estudos (Holmdahl, Sävenstedt \& Imoni, 2014; Picollo \& Fachini, 2019). Adicionalmente, as produções destacam para a necessidade da assistência de enfermagem fazer uso de dialeto compreensível ao paciente e seus familiares, além de fornecer informações relevantes, claras e autorizadas (Stenner, Carey \& Courtenay Graaf, 2016). 
Ainda se tratando do protagonismo das habilidades comunicacionais nos CP no âmbito da APS, destaca-se que dois estudos abarcaram outras intervenções, a saber: avaliar o momento correto para informar más notícias e expressar de forma eficaz sobre questões de fim de vida (Sousa \& Alves, 2015; Graaf, 2016). Sobre a comunicação de notícias difíceis, estudos verificaram que há um distanciamento entre as preferências da pessoa e familiares e como esta de fato acontece na prática clínica (Fan et al., 2019). Além disso, já foi observado que médicos e enfermeiros assumem uma postura de realizar a comunicação de notícias difíceis tardiamente e sem preparo aos envolvidos (Pfeil, Laryionava \& Reiter-Theil, 2015). Entretanto, um dos poucos estudos com pessoas em CP referiram ter suas necessidades atendidas nesse momento de fim de vida, atribuíram este fato a comunicação eficaz com os profissionais, corroborando com os resultados encontrados no presente estudo (Galvão, Borges \& Pinho, 2017).

Do ponto de vista dos profissionais, o envolvimento em comunicação de notícias difíceis agrega confiança nas suas habilidades profissionais (Mishelmovich, Arber \& Odelius, 2016). E que essas discussões quando iniciadas já no âmbito da graduação promovem a superação de medos, a redução de ansiedade e o aumento da confiança diante de situações de terminalidade (Malta, Rodrigues \& Priolli, 2018). Na realidade brasileira já existem protocolos para comunicação de notícias difíceis comprovadamente adequadas, como o "P-A-C-I-E-N-T-E" (Pereira et al., 2017).

Diante do exposto, é possível afirmar que as habilidades comunicacionais são de vital importância para a qualidade da assistência em CP e que o maior impeditivo encontrado, foi uma profissionalização e qualificação inadequados às necessidades observadas na prática clínica.

Entende-se que o artigo traz contribuições para enfermagem nos domínios da formação e da atuação, ao sistematizar e sumarizar uma gama de conhecimentos acerca das intervenções de enfermagem aos pacientes em CP na APS. Essa síntese de evidências favorece a compreensão da abrangência e do escopo das ideias relativas as intervenções, instrumentalizando a prática profissional do enfermeiro na lógica da organização dos CP na APS.

\section{Conclusão}

Esta revisão mapeou as informações sobre as intervenções de enfermagem em CP na APS. Ressalta-se que é uma temática pouco discutida e conhecida pelos profissionais que trabalham no âmbito da APS. Por conseguinte, a partir da análise das intervenções levantadas nos estudos que compuseram a Revisão de Escopo, foi possível perceber a necessidade de aprimoramento profissional quando relacionada aos $\mathrm{CP}$, a fim de aumentar o número de profissionais capacitados na área $\mathrm{e}$ melhorar a qualidade do serviço prestado pela APS. Além disso, a capacitação profissional influencia diretamente nas habilidades comunicacionais e no manejo de sinais e sintomas no domicílio, uma vez que o enfermeiro se torna encarregado de entender as necessidades da pessoa e familiares e instruí-los quanto a possíveis situações a serem enfrentadas.

Os resultados dos estudos incluídos nesta revisão apoiam a importância da efetivação das políticas de saúde que focam a assistência integral, fazendo do enfermeiro um vínculo entre a pessoa, a família e a equipe multiprofissional, visando sempre a avaliação e a melhoria no serviço prestado, com vistas a buscar alternativas como estratégias de comunicação para fortalecer a relação do domić́lio com a APS. Além disso, os estudos destacam a necessidade de olhar para o familiar e o cuidador informal de maneira crítica, visando avaliar a sobrecarga física, emocional e socioeconômica, com o intuito de minimizar o impacto sobre a pessoa.

É esperado que os dados levantados por essa revisão possam agregar à prática cotidiana do enfermeiro para a melhoria dos serviços em CP prestados, visando sempre o bem-estar do indivíduo em CP e de seus familiares. Espera-se também, que as lacunas aqui ressaltadas motivem o desenvolvimento de novos estudos com maior rigor metodológico, visando a produção de evidências científicas confiáveis sobre as intervenções de enfermagem em CP na APS. 


\section{Referências}

Academia Nacional de Cuidados Paliativos. (2020). Atlas de Cuidados Paliativos no Brasil 2019.

Afshar, K., Wiese, B., Schneider, N., \& Müller-Mundt, G. (2020). Systematic identification of critically ill and dying patients in primary care using the German version of the Supportive and Palliative Care Indicators Tool (SPICT-DE). German medical science: GMS e-journal, 18, https://doi.org/10.3205/000278

Almeida, P., Marin, J. \& Casotti, E. (2017). Estratégias Para Consolidação da Coordenação do Cuidado pela Atenção Básica. Trab. educ. Saúde, 15(2):373398. https://doi.org/10.1590/1981-7746-sol00064

Ann-Yi, S., Bruera, E., Wu, J., Liu, D. D., Agosta, M., Williams, J. L., et al. (2018). Characteristics and Outcomes of Psychology Referrals in a Palliative Care Department. Journal of pain and symptom management, 56(3), 344-351. https://doi.org/10.1016/j.jpainsymman.2018.05.022

Arksey, H. \& O'Malley, L. (2005). Scoping studies: towards a methodological framework. International Journal of Social Research Methodology, 8(1):19-32. https://doi.org/10.1080/1364557032000119616

Baer, L. \& Weinstein, E. (2013). Improving oncology nurses' communication skills for difficult conversations. Clinical journal of oncology nursing, 17(3):E45-E51. https://doi.org/10.1188/13.CJON.E45-E51

Barros, M. A. A., Pereira, F. J. R., Abrantes, M. W., Silva, G. B., Porto, V. A. \& Carvalho, M. A. P. (2020). Produção Científica Acerca da Dor em Cuidados Paliativos: Contribuição da Enfermagem no Cenário Brasileiro. Revista Online de Pesquisa Cuidado é Fundamental, 12(1):744-750. http://dx.doi.org/10.9789/2175-5361.rpcfo.v12.9452

Borba, A. S., Ramos, T. M., Rodrigues, G. M. M. \& Lemos, L. R. (2019). Sistematização da assistência de enfermagem a pacientes com distúrbios depressivos. Brazilian Journal of Health Review, 2(6):5217-5227. https://doi.org/10.34119/bjhrv2n6-026

Brant, J., Fink, R. \& Thompson, C. (2019). Global Survey of the Roles, Satisfaction, and Barriers of Home Health Care Nurses on the Provision of Palliative Care. Journal of palliative medicine, 22(8):945-960. https://doi.org/10.1089/jpm.2018.0566

Brasil. Ministério da Saúde; Política Nacional de Atenção Básica, Departamento de Atenção Básica. (2017). Portaria no. 2.436 de 21 de setembro de 2017. Brasília, 2017.

Bulechek, G., Butcher, H. \& Dochterman, J. (2016). NIC Classificação das Intervenções de Enfermagem. (6a ed.).

Campos, S., Zhang, L., Sinclair, E., Tsao, M., Barnes, E. A., Danjoux, C., et al. (2009). The palliative performance scale: examining its inter-rater reliability in an outpatient palliative radiation oncology clinic. Supportive care in cancer: official journal of the Multinational Association of Supportive Care in Cancer, 17(6), 685-690. https://doi.org/10.1007/s00520-008-0524-z

Cleary, A. (2016). Integrating palliative care into primary care for patients with chronic, life-limiting conditions. The Nurse practitioner, 41(3):42-49. https://doi.org/10.1097/01.NPR.0000480588.01667.58

Colquhoun, H., Levac, D., O’Brien, K. K., Straus, S., Tricco, A. C., Perrier, L., et al. (2014). Scoping reviews: Time for clarity indefinition, methods and reporting. Journal of Clinical Epidemiology, 67(12).1291-1294. https://doi.org/10.1016/j.jclinepi.2014.03.013

Coyne, P., Mulvenon, C. \& Paice, J. (2018). American Society for Pain Management Nursing and Hospice and Palliative Nurses Association Position Statement: Pain Management at the End of Life. Pain management nursing: official journal of the American Society of Pain Management Nurses, 19(1):3-7. https://doi.org/10.1016/j.pmn.2017.10.019

Cronin, J. \& Finn, S. (2017). Implementing and evaluating the COMFORT Communication in palliative care curriculum for oncology nurses. J Hosp Palliat Nurs, 19(2017):140-146. 10.1097 / NJH.0000000000000320

Dadalto, L. Testamento Vital. Editora Atlas.

Dahlin, C., Coyne, P. \& Cassel, J. (2016). The Advanced Practice Registered Nurses Palliative Care Externship: A Model for Primary Palliative Care Education. Journal of palliative medicine, 19(7):753-759. https://doi.org/10.1089/jpm.2015.0491

Danielsen, B. V., Sand, A. M., Rosland, J. H., \& Førland, O. (2018). Experiences and challenges of home care nurses and general practitioners in home-based palliative care - a qualitative study. BMC palliative care, 17(1), 95. https://doi.org/10.1186/s12904-018-0350-0

Elk, R., Felder, T. M., Cayir, E., \& Samuel, C. A. (2018). Social Inequalities in Palliative Care for Cancer Patients in the United States: A Structured Review. Seminars in oncology nursing, 34(3), 303-315. https://doi.org/10.1016/j.soncn.2018.06.011

Fan, Z., Chen, L., Meng, L., Jiang, H., Zhao, Q., Zhang, L., \& Fang, C. K. (2019). Preference of cancer patients and family members regarding delivery of bad news and differences in clinical practice among medical staff. Supportive care in cancer: official journal of the Multinational Association of Supportive Care in Cancer, 27(2), 583-589. https://doi.org/10.1007/s00520-018-4348-1

Fitch, M., Fliedner, M. \& O'Connor, M. (2015). Nursing perspectives on palliative care 2015. Annals of palliative medicine, 4(3):150-155. https://doi.org/10.3978/j.issn.2224-5820.2015.07.04

Fox, S., Azman, A. \& Timmons, S. (2020). Palliative care needs in Parkinson's disease: focus on anticipatory grief in family carers. Annals of palliative medicine, 9(1):S34-S43. https://doi.org/10.21037/apm.2020.02.04

Frazzatto, C. \& Sawaia, B. (2016). A critical view of the 'social reinsertion' concept and its implications for the practice of psychologists in the area of mental health in the Brazilian Unified Health System (Sistema Único de Saúde). Journal of health psychology, 21(3):409-418. https://doi.org/10.1177/1359105316628751 
Galvão, M., Borges, M. \& Pinho, D. (2017). Comunicação interpessoal com pacientes oncológicos em cuidados paliativos. Rev baiana enferm, 31(3). http://dx.doi.org/10.18471/rbe.v31i3.22290

Graaf, E. (2016). Hospice assist at home: does the integration of hospice care in primary healthcare support patients to die in their preferred location - A retrospective cross-sectional evaluation study. Palliative Medicine, 30(6):580-586. https://doi.org/10.1177/0269216315626353

Griffiths, J., Ewing, G., Wilson, C., Connolly, M., \& Grande, G. (2015). Breaking bad news about transitions to dying: a qualitative exploration of the role of the District Nurse. Palliative medicine, 29(2), 138-146. https://doi.org/10.1177/0269216314551813

Griffiths, J., Wilson, C., Ewing, G., Connolly, M., \& Grande, G. (2015). Improving communication with palliative care cancer patients at home - A pilot study of SAGE \& THYME communication skills model. European journal of oncology nursing: the official journal of European Oncology Nursing Society, 19(5), 465-472. https://doi.org/10.1016/j.ejon.2015.02.005

Gunnarsdottir, S., Sigurdardottir, V., Kloke, M., Radbruch, L., Sabatowski, R., Kaasa, S., et al. (2017). A multicenter study of attitudinal barriers to cancer pain management. Supportive care in cancer: official journal of the Multinational Association of Supportive Care in Cancer,25(11), 3595-3602. https://doi.org/10.1007/s00520-017-3791-8

Harzheim, E., Pinto, L. F., Hauser, L. \& Soranz, D. (2016). Assessment of child and adult users of the degree of orientation of Primary Healthcare in the city of Rio de Janeiro, Brazil. Ciência \& Saúde Coletiva, 21(5):1399-1408. https://dx.doi.org/10.1590/1413-81232015215.26672015

Hawkins, K., Parker, P. M., Hommer, C. E., Bhattarai, G. R., Huang, J., Wells, T. S., Ozminkowski, R. J., \& Yeh, C. S. (2015). Evaluation of a high-risk case management pilot program for Medicare beneficiaries with Medigap coverage. Population health management, 18(2), 93-103. https://doi.org/10.1089/pop.2014.0035

Hernando, C., Limonero, J. \& Gil, F. (2020). Psychological intervention in patients with advanced cancer at home through Individual Meaning-Centered Psychotherapy-Palliative Care: a pilot study. Supportive care in cancer: official journal of the Multinational Association of Supportive Care in Cancer, 28(10):4803-4811. https://doi.org/10.1007/s00520-020-05322-2

Holmdahl, S., Sävenstedt, S. \& Imoni, R. (2014). Parenteral nutrition in home-based palliative care: Swedish district nurses experiences. Scandinavian journal of caring sciences, 28(1):89-96. https://doi.org/10.1111/scs.12038

Hudson, B. F., Best, S., Stone, P., \& Noble, T. B. (2019). Impact of informational and relational continuity for people with palliative care needs: a mixed methods rapid review. BMJ open, 9(5), e027323. https://doi.org/10.1136/bmjopen-2018-027323

Hweidi, I. M., Tawalbeh, L. I., Al-Hassan, M. A., Alayadeh, R. M., \& Al-Smadi, A. M. (2017). Research Use of Nurses Working in the Critical Care Units: Barriers and Facilitators. Dimensions of critical care nursing: DCCN, 36(4), 226-233. https://doi.org/10.1097/DCC.0000000000000255

Ikeda, L., Marcheti, M., Sales, A., Giacon, B. \& Marques, F. (2017). Dificuldades de uma equipe de enfermagem para prestar cuidados paliativos. CIAIQ 2(1):732-741

Karnick, P. M. (2016). Evidence-Based Practice and Nursing Theory. Nursing science quarterly, 29(4):283-284. https://doi.org/10.1177/0894318416661107

Knaul, F. M., Farmer, P. E., Krakauer, E. L., De Lima, L., Bhadelia, A., Jiang Kwete, X., et al. Lancet Commission on Palliative Care and Pain Relief Study Group (2018). Alleviating the access abyss in palliative care and pain relief-an imperative of universal health coverage: the Lancet Commission report. Lancet (London, England), 391(10128), 1391-1454. https://doi.org/10.1016/S0140-6736(17)32513-8

Lai, X., Wong, F. \& Ching S. (2018). The experience of caring for patients at the end-of-life stage in non-palliative care settings: a qualitative study. BMC palliative care, 17(1):116. https://doi.org/10.1186/s12904-018-0372-7

Levac, D., Colquhoun, H. \& O'Brien, K. (2010). Scoping studies: advancing the methodology. Implementation science: IS, 5:69. https://doi.org/10.1186/17485908-5-69

Levine, S., O'Mahony, S., Baron, A., Ansari, A., Deamant, C., Frader, J., et al. (2017). Training the Workforce: Description of a Longitudinal Interdisciplinary Education and Mentoring Program in Palliative Care. Journal of pain and symptom management, 53(4), 728-737. https://doi.org/10.1016/j.jpainsymman.2016.11.009

Lokker, M. E., Swart, S. J., Rietjens, J., Van Zuylen, L., Perez, R., \& van der Heide, A. (2018). Palliative sedation and moral distress: A qualitative study of nurses. Applied nursing research: ANR, 40, 157-161. https://doi.org/10.1016/j.apnr.2018.02.002

Malta, R., Rodrigues, B. \& Priolli, D. (2018). Paradigma da formação Médica: Atitudes e Conhecimentos de Acadêmicos sobre a Morte e Cuidados Paliativos. Rev. Brasileira de Educação Médica, 42(2):34-44. https://doi.org/10.1590/1981-52712015v42n2rb20170011.

Marcucci, F. C., Cabrera, M. A., Perilla, A. B., Brun, M. M., de Barros, E. M., Martins, V. M., et al. (2016). Identification and characteristics of patients with palliative care needs in Brazilian primary care. BMC palliative care, 15, 51. https://doi.org/10.1186/s12904-016-0125-4

Mayahara, M., Foreman, M. D., Wilbur, J., Paice, J. A., \& Fogg, L. F. (2015). Effect of hospice nonprofessional caregiver barriers to pain management on adherence to analgesic administration recommendations and patient outcomes. Pain management nursing: official journal of the American Society of Pain Management Nurses, 16(3), 249-256. https://doi.org/10.1016/j.pmn.2014.07.001

Meier, E. A., Gallegos, J. V., Thomas, L. P., Depp, C. A., Irwin, S. A., \& Jeste, D. V. (2016). Defining a Good Death (Successful Dying): Literature Review and a Call for Research and Public Dialogue. The American journal of geriatric psychiatry: official journal of the American Association for Geriatric Psychiatry, 24(4), 261-271. https://doi.org/10.1016/j.jagp.2016.01.135

Mertens, F., De Gendt, A., Deveugele, M., Van Hecke, A., \& Pype, P. (2019). Interprofessional collaboration within fluid teams: Community nurses' experiences with palliative home care. Journal of clinical nursing, 28(19-20), 3680-3690. https://doi.org/10.1111/jocn.14969 
Midtbust, M. H., Alnes, R. E., Gjengedal, E., \& Lykkeslet, E. (2018). Perceived barriers and facilitators in providing palliative care for people with severe dementia: the healthcare professionals' experiences. BMC health services research, 18(1), 709. https://doi.org/10.1186/s12913-018-3515-x

Mishelmovich, N., Arber, A. \& Odelius, A. (2016). Breaking significant news: The experience of clinical nurse specialists in cancer and palliative care. European journal of oncology nursing: the official journal of European Oncology Nursing Society, 21:153-159. https://doi.org/10.1016/j.ejon.2015.09.006

Nevado, D., Babarro, A. \& Lozano, M. (2012). Continuidad de cuidados, innovación y redefinición de papeles profesionales en la atención a pacientes crónicos y terminales. Informe SESPAS, Gaceta Sanitaria, 24(1):63-68. https://doi.org/10.1016/j.gaceta.2011.09.032

Omondi, S., Weru, J., Shaikh, A. J., \& Yonga, G. (2017). Factors that influence advance directives completion amongst terminally ill patients at a tertiary hospital in Kenya. BMC palliative care, 16(1), 9. https://doi.org/10.1186/s12904-017-0186-z

Paim, J. (2018). Sistema Único de Saúde (SUS) aos 30 anos. Ciência \& Saúde Coletiva, 23(6):1723-1728. https://dx.doi.org/10.1590/141381232018236.09172018

Pereira, D. G., Fernandes, J., Ferreira, L. S., Rabelo, R. O., Pessalacia, J. D. R. \& Souza, R. S. (2017). Significados dos cuidados paliativos na ótica de enfermeiros e gestores da atenção primária à saúde. Revista de Enfermagem UFPE, 11(3):1357-1364. https://doi.org/10.5205/1981-8963-v11i3a13977p1357$1364-2017$

Peters, M. D. J., Godfrey, C., McInerney, P., Munn, Z., Tricco, A. C. \& Khalil, H. (2020). Capítulo 11: Revisões do escopo (versão 2020). In: Aromataris, E. \& Munn Z (Editores). JBI Manual for Evidence Synthesis, JBI. https://doi.org/10.46658/JBIMES-20-12

Pfeil, T., Laryionava, K. \& Reiter-Theil, S. (2015). What keeps oncologists from addressing palliative care early on with incurable cancer patients? An active stance seems key. The oncologist, 20(1):56-61. https://doi.org/10.1634/theoncologist.2014-0031

Picollo, D. \& Fachini, M. (2018). A atenção do enfermeiro ao paciente em cuidado paliativo. Rev Ciênc Med, 27(2):85-92. http://dx.doi.org/10.24220/2318$0897 \mathrm{v} 27 \mathrm{n} 2 \mathrm{a} 3855$

Pirret, A., Neville, S. \& La Grow, S. (2015). Nurse practitioners versus doctors diagnostic reasoning in a complex case presentation to an acute tertiary hospital: a comparative study. International journal of nursing studies, 52(3):716-726. https://doi.org/10.1016/j.ijnurstu.2014.08.009

Portela, G. (2017). Atenção Primária à Saúde: um ensaio sobre conceitos aplicados aos estudos nacionais. Physis: Revista de saúde coletiva, 27(2):255-176. https://doi.org/10.1590/s0103-73312017000200005

Ribeiro, J. \& Poles, K. (2019). Cuidados Paliativos: Prática dos Médicos da Estratégia Saúde da Família. Rev. Bras. Educ. Med., 43(3):62-72. https://doi.org/10.1590/1981-52712015v43n3rb20180172

Roth, A. \& Canedo, A. (2019). Introduction to Hospice and Palliative Care. Primary care, 46(3):287-302. https://10.1016/j.pop.2019.04.001

Silva, M. (2013). O papel do profissional da Atenção Primária à Saúde em cuidados paliativos. Revista Brasileira de Medicina de Família e Comunidade, 9(30):45-53. https://doi.org/10.5712/rbmfc9(30)718

Sousa, J. \& Alves, E. (2015). Competências do enfermeiro para o cuidado paliativo na atenção domiciliar. Acta Paulista de Enfermagem, 28(3):264-269. https://doi.org/10.1590/1982-0194201500044

Srivastava, P. \& Hopwood, N. (2009). A practical iterative framework for qualitative data analysis. Int J Qual Methods, 8(1):76-84. https://doi.org/10.1177/160940690900800107

Stenner, K., Carey, N. \& Courtenay, M. (2012). Prescribing for pain--how do nurses contribute? A national questionnaire survey. Journal of clinical nursing, 21(23-24):3335-3345. https://doi.org/10.1111/j.1365-2702.2012.04136.x

Tornøe, K., Danbolt, L. J., Kvigne, K., \& Sørlie, V. (2015). A mobile hospice nurse teaching team's experience: training care workers in spiritual and existential care for the dying - a qualitative study. BMC palliative care, 14, 43. https://doi.org/10.1186/s12904-015-0042-y

Tricco, A. C., Lillie, E., Zarin, W., O'Brien, K. K., Colquhoun, H., Levac, et al. (2018). PRISMA Extension for Scoping Reviews (PRISMA-ScR): Checklist and Explanation. Annals of internal medicine, 169(7), 467-473. https://doi.org/10.7326/M18-0850

Ullrich, A., Ascherfeld, L, Marx, G., Bokemeyer, C., Bergelt, C. \& Oechsle, K. (2017). Quality of life, psychological burden, needs, and satisfaction during specialized inpatient palliative care in family caregivers of advanced cancer patients. BMC palliative care, 16(1):31. https://doi.org/10.1186/s12904-017-0206$\mathrm{z}$

Van den Brekel, L., Van der Baan, F. H., Zweers, D., Koldenhof, J. J., Vos, J., de Graeff, A., et al. (2020). Predicting Anxiety in Hospitalized Cancer Patients. Journal of pain and symptom management, 60(3), 522-530.e1. https://doi.org/10.1016/j.jpainsymman.2020.04.005

Van der Plas, A. G., Onwuteaka-Philipsen, B. D., Vissers, K. C., Deliens, L., Jansen, W. J., \& Francke, A. L. (2016). Appraisal of cooperation with a palliative care case manager by general practitioners and community nurses: a cross-sectional questionnaire study. Journal of advanced nursing, 72(1), 147-157. https://doi.org/10.1111/jan.12818

Virdun, C., Luckett, T., Lorenz, K., Davidson, P. M., \& Phillips, J. (2017). Dying in the hospital setting: A meta-synthesis identifying the elements of end-oflife care that patients and their families describe as being important. Palliative medicine, 31(7), 587-601. https://doi.org/10.1177/0269216316673547

Warth, M., Kessler, J., Koehler, F., Aguilar-Raab, C., Bardenheuer, H. J., \& Ditzen, B. (2019). Brief psychosocial interventions improve quality of life of patients receiving palliative care: A systematic review and meta-analysis. Palliative medicine, 33(3), 332-345. https://doi.org/10.1177/0269216318818011

Watts, T. (2014). Secondary breast cancer: the role of primary care nurses. Practice Nurse. 44(5):36-41. 
Research, Society and Development, v. 10, n. 8, e24710817365, 2021

(CC BY 4.0) | ISSN 2525-3409 | DOI: http://dx.doi.org/10.33448/rsd-v10i8.17365

Wenger, N. S., Roth, C. P., Martin, D., Nickels, L., Beckman, R., Kamberg, C., et al. (2011). Quality of Care Provided in a Special Needs Plan Using a Nurse Care Manager Model. Journal of the American Geriatrics Society, 59(10):1810-1822. https://doi.org/10.1111/j.1532-5415.2011.03599.x

Wittenberg, E. \& Goldsmith J. (2016). The COMFORT Communication Project. 